\title{
VARIA
}

\section{A PROPÓSITO DE ALGUNOS «CAPRICHOS» DE GOYA Y UN «EXEMPLARIO» ARAGONÉS DE 1531}

Ante la abundante bibliografía sobre Goya, especialmente la referida al significado y fuentes de inspiración de sus obras, produce pudor insistir en este sentido, sobre todo teniendo en cuenta la originalidad del pintor ${ }^{1}$.

En alguna ocasión he llamado la atención sobre la influencia formal del Bosco en algunos grabados de Goya ${ }^{2}$, hoy me atrevo a sugerir la posible inspiración que pudieron tener en él algunos de los grabados que ilustran el Exemplario contra los engaños y peligros del mundo, publicado en Zaragoza en 1531, y que sin duda conoció el pintor (fig. 1).

Si observamos el «Capricho» titulado Tú que no puedes, vemos que en él aparecen dos hombres cargados con asnos (fig. 2). Una imagen similar se halla en el Exemplario, con la diferencia de que esta vez es un fraile el que soporta sobre sus espaldas a una cabra, titulándose: La mentira de muchos muchas veces tiene lugar de verdad (fig. 3). Pérez Sánchez — seguido por Flecha- interpretaron la escena de Goya como la carga que soportaban los campesinos teniendo que mantener con su trabajo y sacrificio los privilegios de los poderosos: nobleza y clero $^{3}$. López Vázquez, la puso en relación con un emblema de Faxardo, en el que se resalta la fuerza del sufrimiento ${ }^{4}$.

Desde luego ninguna de estas interpretaciones tiene nada que ver con el mensaje del texto que acompaña al grabado del Exemplario, en el que se resalta el carácter simplón del fraile engañado por los que le rodean y le dicen que lo que lleva sobre sus espaldas es un perro, no una cabra, llegándoselo a creer ${ }^{5}$. Teniendo en cuenta el carácter y significado del asno como imagen de la ignorancia —incluso en la misma obra de Goya-, tal vez el pintor enfatizó el texto e imagen del Exemplario, colocando sobre dos «simplones» el asno de la ignorancia, en lugar de la cabra.

Más evidente, en cuanto al contenido y la forma, es el paralelismo existente entre el $\mathrm{Ca}$ pricho 53: «Que pico de oro», en el que un papagayo habla a un grupo de frailes (fig. 4), y el

\footnotetext{
${ }^{1}$ A. E. Pérez Sánchez, Goya. Caprichos, Desastres, Tauromaquia, Disparates. Madrid, 1979; López Vázquez, Los Caprichos de Goya. Santiago de Compostela, 1982; Flecha, Literatura e Ideología en el arte de Goya. Zaragoza, 1988; J. F. Esteban Lorente, «Jeroglíficos, Alegorías y Emblemas en Goya», Artigrama, 2003, pág. 471-503.

${ }^{2}$ I. Mateo Gómez, El Bosco en España. Madrid, 1991 ( $2^{\mathrm{a}} \mathrm{ed}$.); «De nuevo Goya y el Bosco», Archivo Español de Arte, 1995, pág. 60-61.

${ }^{3}$ Pérez Sánchez, Flecha, loc. cit. nota 1.

${ }^{4}$ López Vázquez, loc. cit. nota 1.

${ }^{5}$ Exemplario, $\mathrm{f}^{\circ}$ LIII $^{\mathrm{v}}$

${ }^{6}$ López Vázquez, Flecha, loc. cit. nota 1.
} 

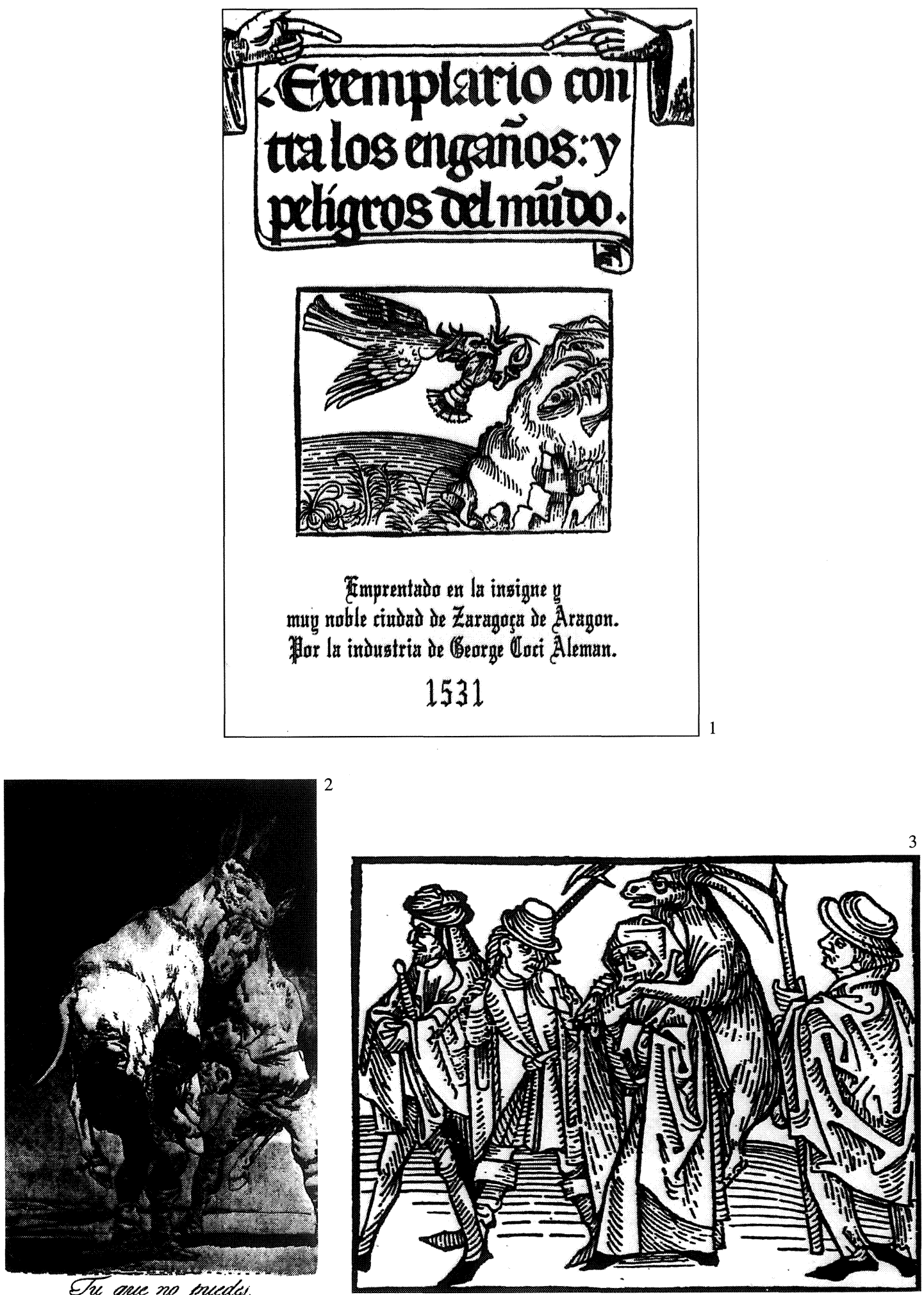

Fig. 1. Portada del Exemplario, publicado en Zaragoza en 1531

Fig. 2. Goya, Capricho. «Tú que no puedes».

Fig. 3. Exemplario (f. ${ }^{\text {os }}$ LIII-LIII ${ }^{v}$ ) «La mentira de muchos muchas veces tiene lugar de verdad». 


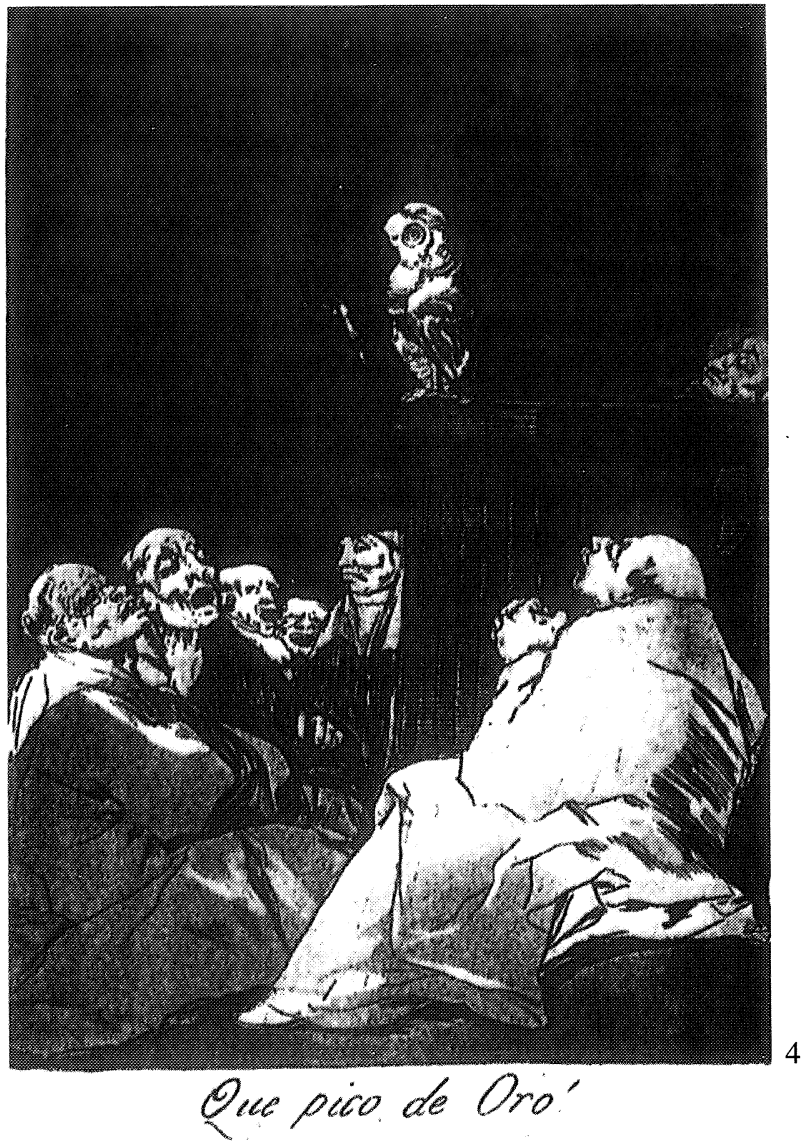

5

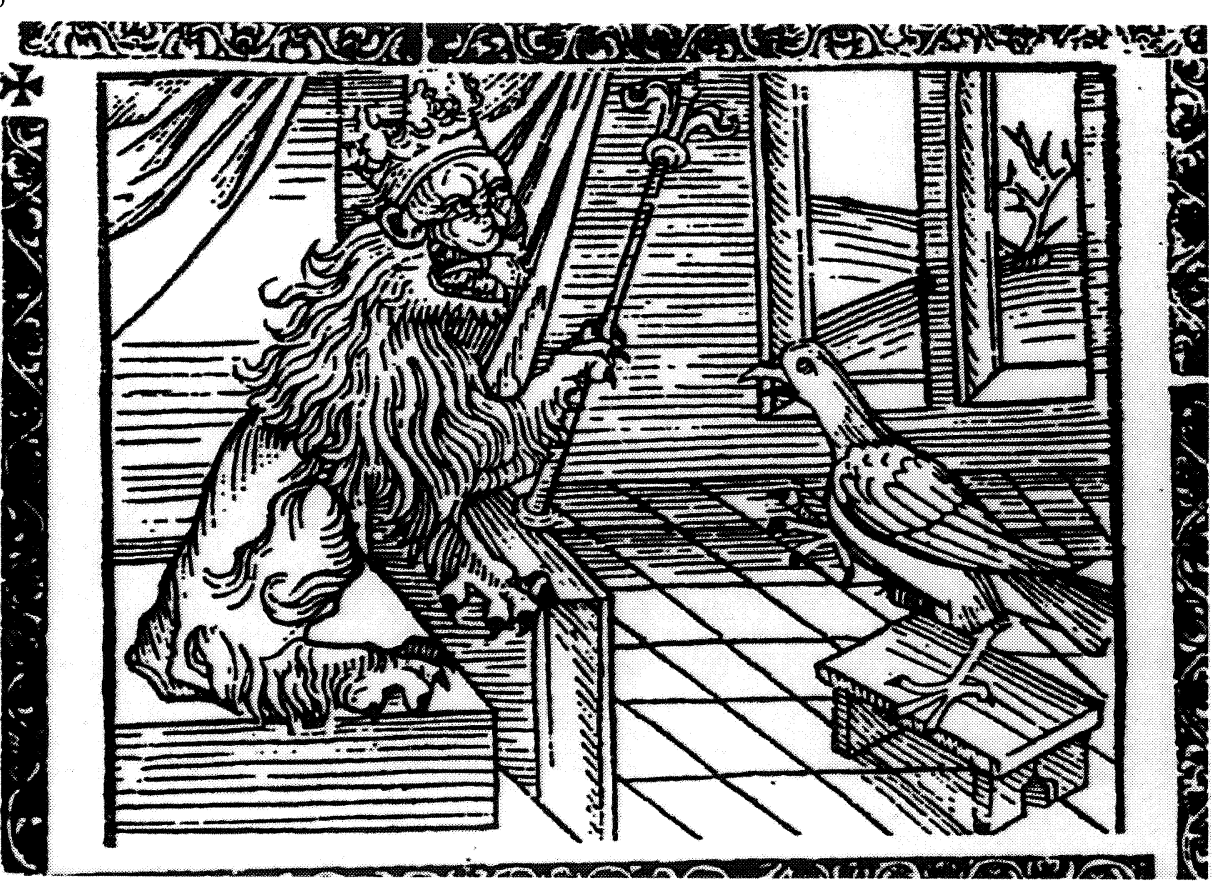

Fig. 4. Goya, Capricho. «Qué pico de oro».

Fig. 5. Exemplario (f. $\left.{ }^{\circ} \mathrm{XIV}^{v}\right)$. «Las palabras vanas y malignas desvían el santo propósito» 

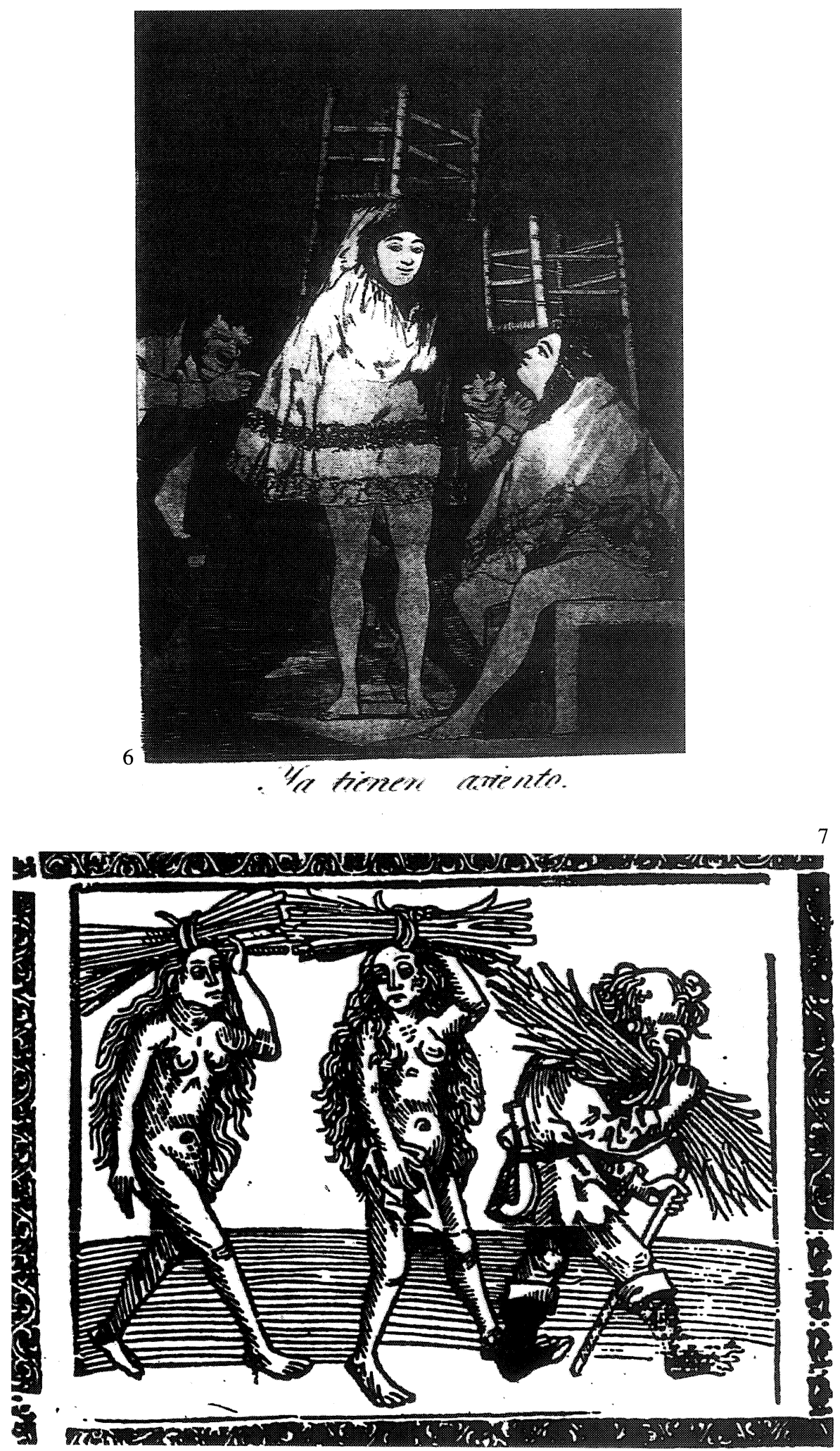

Fig. 6. Goya, Capricho. «Ya tienen asiento».

Fig. 7. Exemplario (f. ${ }^{\circ}$ XXXIII). "Oye sus defectos el que no quiere callar los ajenos». 
grabado del Exemplario donde es un león el que escucha a un cuervo (fig. 5). El grabado de Goya ha sido interpretado por López Vázquez y Flecha, como una degradación de la oratoria sagrada, y en el Exemplario se advierte sobre las palabras vanas y malignas que desvían «el santo propósito» ${ }^{7}$.

Cierta similitud hallamos entre el Capricho 26 de Goya: «Ya tienen asiento», en el que unas mujeres portan sobre sus cabezas sillas de madera con asientos de anea o paja (fig. 6), y un grabado del Exemplario titulado: «Oye sus defectos el que no quiere callar los ajenos», en el que dos mujeres llevan sobre sus cabezas gavillas de anea o madera (fig. 7). Pérez Sánchez interpretó el grabado goyesco como una referencia a aquellas mujeres que se procuran «asiento», no precisamente por medio de sus buenas costumbres y López Vázquez lo pone en relación con un emblema de Faxardo en el que se alude a los «serviles» ante la amenaza y el castigo, más que ante el ruego. En ambas historias las mujeres se hallan acompañadas por hombres que las acusan de su actitud, ridiculizándola o moralizando sobre ella.

De todo lo que acabamos de comentar tal vez se pueda deducir que Goya pudo inspirarse, formalmente, en algunos grabados del Exemplario publicado en su ciudad natal, y que tanto el texto de este último, como la intención de fondo de Goya en sus Caprichos, son una crítica a la sociedad expresada según la sensibilidad artística de cada autor.

De otro lado la sátira de las obras humanas a través de la literatura fabulística tiene un largo y prestigioso recorrido desde la antigüedad, existiendo de ella abundantes ediciones en épocas posteriores. Recordemos el éxito en el siglo XVII de las fábulas de La Fontaine, y más tarde aquellas con las que convivió Goya escritas por Iriarte y Samaniego. El contacto de Goya con este mundo no se puede negar, pues si nos fijamos en algunos títulos de Iriarte parece que estamos leyendo epígrafes de Goya para sus Caprichos: «Hacer que hacemos», «Donde las dan las toman», «El asno erudito», «Pena casos tales», etc. ${ }^{9}$.

Hedith Helman señala como Goya se propuso en los Caprichos la censura de los errores y vicios humanos de la sociedad que le tocó vivir y que hasta entonces sólo la literatura había tenido el privilegio de reflejar ${ }^{10}$. Lo que hay que subrayar, también, es que tanto Goya, en sus Caprichos, como Iriarte en sus Fábulas Literarias y Poesías, instituyen unas preceptivas literarias y artísticas más acordes con la crítica ridiculizadora y burlesca de La Fontaine, que con el carácter didáctico-moralizador de la fábula antigua y del Exemplario de 1531.

Que Goya conoció la obra de Iriarte es evidente, pero ¿dónde pudo conocer el Exemplario contra los engaños y peligro del mundo? En primer lugar, al estar editado en Zaragoza, pudo conocerlo en cualquier biblioteca de la ciudad, e incluso tener un ejemplar propio. También pudo manejarlo en casa de Iriarte, quien debía de tener abundante bibliografía sobre el tema. No debemos olvidar tampoco que posiblemente la biblioteca de Iriarte pudo enriquecerse con volúmenes de la de su tio Juan de Iriarte, en la que, entre otras obras, había más de dos mil refranes —en dos o tres formas- y más de mil epigramas en latín y castellano.

Isabel Mateo GÓMEZ

Dpto. de Historia del Arte. C.S.I.C.

\footnotetext{
${ }^{7}$ Exemplario, $\mathrm{f}^{\mathrm{X}} \mathrm{XXIV}^{\mathrm{v}}$

${ }^{8}$ Exemplario, $\mathrm{f}^{\circ} \mathrm{XXXIII}$

${ }^{9}$ Para las obras de Iriarte y Samaniego se ha consultado E. Cotarelo y Mori, Iriarte y su época, Madrid, 1897 y F. Aguilar Piñol, Bibliografía de autores españoles del s. xVIII. Tomo V. Madrid, 1986.

${ }^{10}$ El trasmundo de Goya. Madrid, 1963. Cita además de la intencionalidad de los fabulistas, la del Padre Feijoo en el Teatro Crítico, y la de Moratín en su Comedia Nueva. Todos ellos, incluido Goya, tuvieron cierta aprensión sobre la suerte que corrían sus obras y ellos mismos al atacar en ellas a los estamentos del poder laico y religioso.
} 